\title{
NUMERICAL ANALYSIS OF THE INFLUENCE OF LATERAL SUSPENSION PARAMETERS ON THE RIDE QUALITY OF RAILWAY VEHICLES
}

\author{
MĂDĂLINA DUMITRIU \\ Politehnica University of Bucharest, Department of Railway Vehicles, Romania \\ e-mail: madalinadumitriu@yahoo.com
}

\begin{abstract}
This paper deals with the influence of parameters of the lateral suspension on the ride quality of railway vehicles during running on a track with lateral deviations, compared to the designing geometry. The ride quality is evaluated at speed $200 \mathrm{~km} / \mathrm{h}$, based on lateral accelerations in three reference points of the vehicle carbody. The values corresponding to these accelerations are derived from numerical simulations on a non-linear model of the vehicle with 21 degrees of freedom. The results thus obtained validate a series of features in vibrational behaviour of the railway vehicle and the possibility to improve the ride quality by the best possible selection of the suspension parameters.
\end{abstract}

Keywords: railway vehicle, ride quality, lateral suspension, vibration behaviour, R.M.S. acceleration

\section{Introduction}

During running, a railway vehicle is subjected to permanent vibrations that can be limited by the suspension to a level that would not affect the dynamic performance of the vehicle. The ability of the vehicle to comply with the transport requirements in terms of the level of vibrations to which they can be subjected, and are dependent on the vehicle type, passengers, goods or the locomotive staff, is assigned a high value by the ride quality (Garg and Dukkipati, 1984). This criterion is considered when evaluating the dynamic behaviour of the railway vehicle for the homologation and its admission into international traffic, besides safety and track fatigue (UIC 518 Leaflet, 2009).

From the perspective of ride quality, the evaluation of dynamic behaviour of railway vehicles mainly involves finding solutions to certain issues of vibrations, which can be dealt with experimentally and theoretically. As a matter of fact, it is about the determination of the acceleration value at the vehicle carbody level, during either line testing (Dumitriu, 2014) or in a virtual environment, based on a numerical model of the vehicle (Sharma, 2011). Numerical simulations are useful tools to estimate the dynamic behaviour of the railway vehicle and to optimize its dynamic performance even at the designing stage, followed by the investigation of the issues emerging during exploitation. In comparison with the line testing that is costly and requires considerable investment of time and effort and may be affected by out-of-control series of variables, numerical simulations have a certain advantage as they allow the examination of the dynamic behaviour of the vehicle even in the vicinity of extreme circumstances that cannot be marked out during real testing conditions (Evans and Berg, 2009; Schupp, 2003).

The numerical evaluation of ride quality is possible via software applications developed on the basis of certain vehicle/track system models. During the modelling stage, which needs to be considered, the railway vehicle represents a complex oscillating system that vibrates both in the vertical and lateral directions. Nevertheless, due to the fact that the vehicle construction generally complies with the rules of geometrical symmetry, both inertial and elastic, vertical motions can be regarded as uncoupled from the lateral ones, hence separately studied. 
As for the lateral vibrations, they are a consequence of the fact that rolling surfaces of the wheels, rigidly fixed on the wheelset, have inversed conicities and are maintained by track lateral irregularities (Iwnicki, 2006). The lateral vibrations are of particular importance, both for vehicle stability and for ensuring ride quality and comfort of passengers. Indeed, the essential features of such vibrations is the tendency to become unstable when the velocity goes beyond a certain value - the critical speed, and unstable running of the vehicle can lead to extreme situations vehicle derailment and track damage. This is the reason why most studies have been focused on the issue of vehicle stability dealt with from different perspectives - methods of stability evaluation (Polach and Kaiser, 2012; Polach, 2006), stability optimization (Mazzola et al., 2010; He and McPhee, 2002), influence of suspension parameters and wheel/rail contact conditions upon the critical speed (Dabin et al., 2012; Huang et al., 2013; Lee and Cheng, 2005; Serajian, 2013; Park et al., 2011), analysis of the stability in curving (Cheng et al., 2009; Zboinski and Dusza, 2010, 2011).

The paper talks about the issue of ride quality in comparison with lateral vibrations of the vehicle, in terms of analysis of the influence of the primary and secondary suspension (stiffness and lateral damping) upon lateral acceleration at the level of the vehicle carbody. This analysis aims to bring a quality identification of the solutions by which the carbody lateral acceleration can be minimised, thus giving the vehicle the best dynamic performance in terms of ride quality. It is about a problem that, to the best knowledge of the author, has not been yet approached from this viewpoint. A literature survey only mentions sensitivity analysis that evaluates the effect of elastic properties in the suspension of the railway vehicles upon the dynamic behaviour of the vehicle regarding safety, track fatigue, ride quality (Suarez et al., 2013). On the whole, eight different elastic and damping properties were considered, excluding lateral damping of the primary suspension.

To reach the goal of this paper, a non-linear complex model with 21 degrees of freedom of the vehicle/track system is recommended. It allows examination of vehicle vibrations during circulation on a track with lateral irregularities. The non-linear nature of the model comes from wheel/rail creep forces, lateral reaction of the rail acting on the wheelset when its clearance on the track is consumed and the load is transferred between the wheels of each wheelset.

To calculate the wheel/rail creep forces, the non-linear Polach model is applied (Polach, 1999) that is herein extended by introducing the influence of the load transfer between the wheels of the same wheelset upon creep coefficients. The results derived from numerical simulations validate the possibility to improve ride quality by a good selection of lateral suspension parameters lateral damping of the secondary suspension or lateral stiffness of the primary suspension.

\section{The model of the vehicle/track system}

Figures 1 and 2 feature the mechanical model of the vehicle/track system herein used for numerical evaluation of ride quality of a railway vehicle during running at velocity $V$ on a track with lateral irregularities. The vehicle is represented with a non-linear mechanical model of 21-degrees of freedom, comprising of 7 rigid bodies representing the carbody, suspended masses of the bogies (the bogies' chassis) as well as four wheelsets connected via Kelvin-Voigt type elements by which the elastic and damping elements of the two suspension levels are modelled.

The vehicle carbody is described by a rigid body with three degrees of freedom and the following motions: lateral $y_{c}$, roll $\varphi_{c}$ and yaw $\alpha_{c}$. The bogie chassis is also modelled as a threedegrees of freedom rigid body, namely lateral $y_{b i}$, roll $\varphi_{b i}$ and yaw $\alpha_{b i}$, with $i=1$ or 2 . It should be noted that $2 a_{c}$ means the vehicle wheelbase and $2 a_{b}$ - the bogie wheelbase.

As for the wheelsets, they are considered to be able to perform the following independent motions: lateral translation $y_{w j,(j+1)}$ and rotation around the vertical wheelset - yaw $\alpha_{w j, j+1}$, 


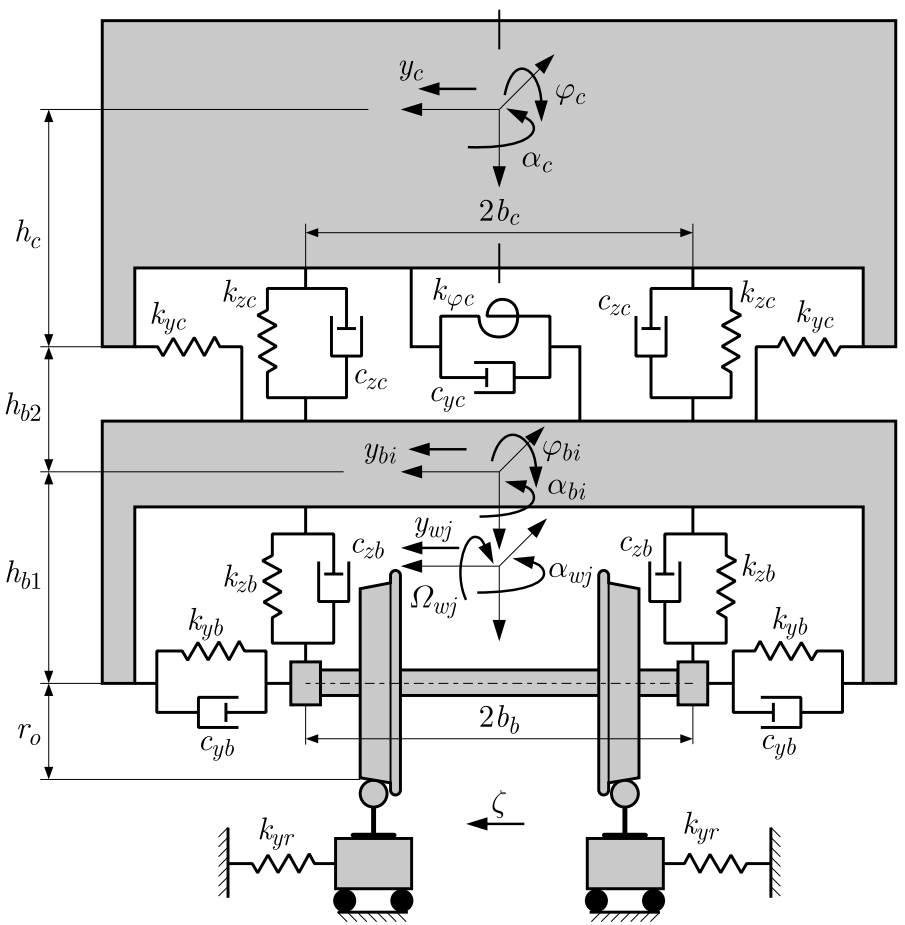

Fig. 1. The mechanical model of the vehicle/track system - front view

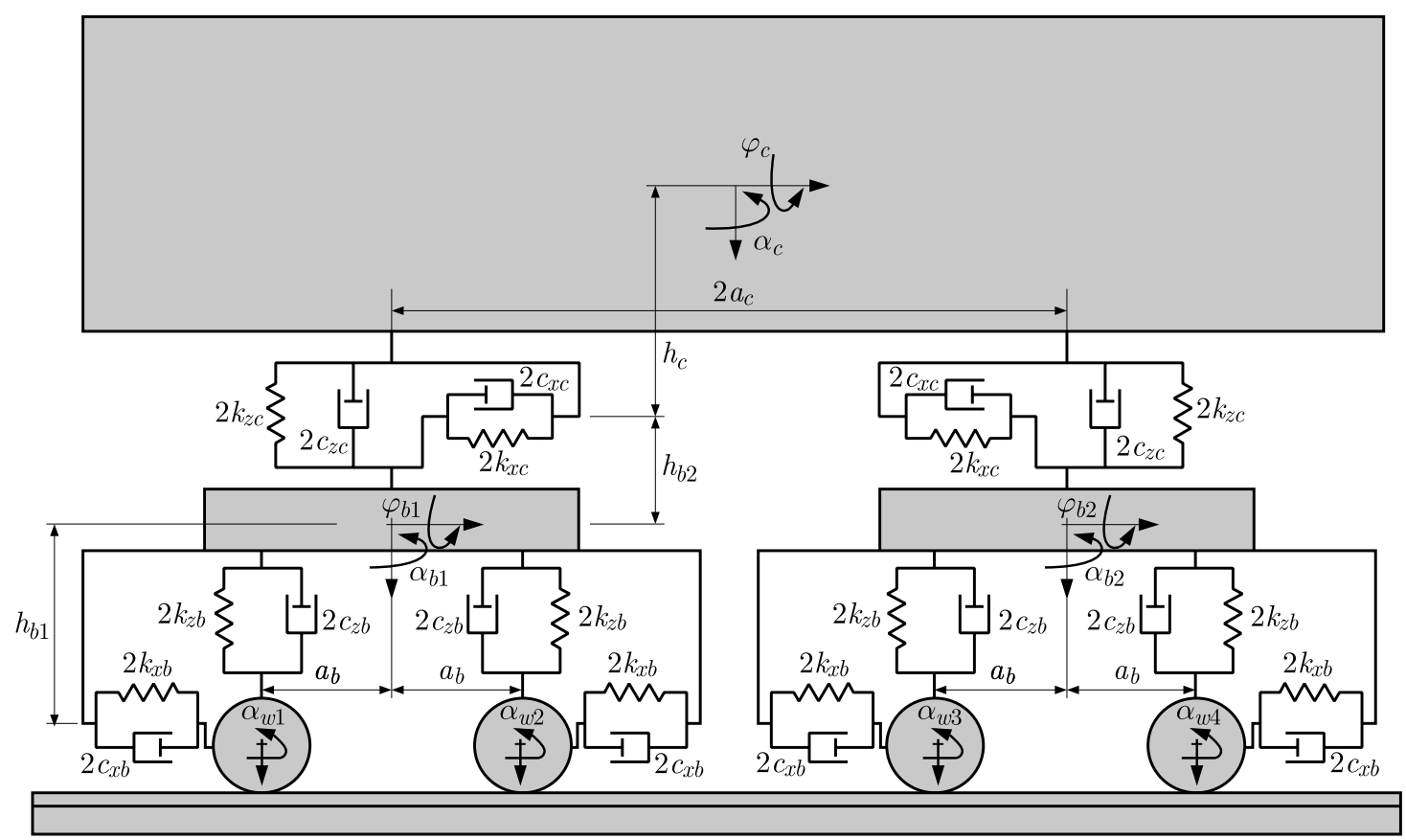

Fig. 2. The mechanical model of the vehicle/track system - side view

where $j=2 i-1$, with $i=1$ or 2 , having in mind that the bogie $i$ has the wheelsets $j$ and $j+1$. Similarly, the wheelset rotates around its own axis at the angular speed $\Omega_{w j,(j+1)}=V / r_{o}+$ $\omega_{w j, j+1}$, where $\omega_{w j, j+1}$ is angular sliding speed of the wheelset compared to $V / r_{o}$, and $r_{o}$ is radius of the rolling circle when the wheelset occupies the median position on the track. Likewise, due to shape of the rolling profiles, the wheelset performs two more motions, namely rolling and bouncing, which are not independent, but affect the wheelset lateral motion on the track. 
The elastic elements of the secondary suspension can be deformed after vertical, lateral and longitudinal directions and they have the rigidities of $k_{z c}, k_{y c}$ and $k_{x c}$. In order to restrict the roll, each bogie has an anti-roll torsion bar system whose stiffness is $k_{\varphi c}$. In the vertical direction, the secondary suspension of the bogie has two dampers with the damping constant $c_{z c}$, while in the horizontal direction there is only one damper with the constant $c_{y c}$. The anti-hunting dampers that are mounted on the lateral sides of the bogies have the damping constant $c_{x c}$. It is mentioned that the plane of the secondary suspension is at height $h_{c}$ referred to the carbody mass centre and at height $h_{b 2}$ referred to the bogie mass centre, while the lateral base of the secondary suspension is $2 b_{c}$. The elements of the primary suspension whose lateral base is $2 b_{c}$ also operate in the three directions and have rigidities $k_{x b}, k_{y b}, k_{z b}$ and damping constants $c_{x b}$, $c_{y b}$ and $c_{z b}$. The Kelvin-Voigt systems located in the axles plane at height $h_{b 1}$ referred to the bogie mass centre are modelling their elastic driving.

As for the track lateral irregularities, they are described by a pseudo-stochastic function $\zeta(x)$, written as

$$
\zeta(x)=\sum_{k=0}^{N} Z_{k} \cos \left(\Omega_{k}+\varphi_{k}\right)
$$

where $Z_{k}$ is the amplitude of the spectral component corresponding to the wave number $\Omega_{k}$, and $\varphi_{k}$ is the lag of the spectral component $k^{\prime}$ for which a uniform random distribution is selected. The amplitude of each spectral component is established on the basis of the power spectral density of the track irregularities described in accordance with ORE B176 and specifications included in the UIC 518 Leaflet regarding the track geometrical quality.

Against each wheelset, the track lateral irregularities are described by the function $\zeta_{j,(j+1)}\left(x_{j,(j+1)}\right)$ dependent on the distance along the track, such as

$$
\zeta_{j, j+1}\left(x_{j, j+1}\right)=\left\{\begin{array}{lll}
0 & \text { for } & x_{j, j+1} \leqslant 0 \\
\zeta\left(x_{j, j+1}\right) & \text { for } & x_{j, j+1}>0
\end{array}\right.
$$

where, depending on the wheelset position in the vehicle unit, $x_{j, j+1}$ is as below:

- for $i=1$

$$
x_{1}=x \quad x_{2}=x-a_{b}
$$

- for $i=2$

$$
x_{3}=x-2 a_{c} \quad x_{4}=x-2 a_{b}-2 a_{c}
$$

where the abscissa $x$ is calculated as a function of the time moment $t, x=V t$.

During circulation on a tangent track, the wheelsets usually follow the trajectory given by the track alignment without consuming the clearance on the track. This occurs when the vehicle exhibits stable behaviour. Under such circumstances, the wheel/rail contact takes place on the rolling surface of the profiles and the contact geometry can be described by linear relations, as for the S78 wheel profile (used in C.F.R. - the Romanian Railway Tracks) and for the UIC 60 rail profile (Dumitriu, 2013). However, there are exceptional situations, such as the loss of stability or the existence of an isolated defect of great amplitude, when it is possible to consume the wheelset clearance on the track and shocks between the wheel flange and the inside rail flank. The modelling of these latter situations is possible by the introduction of a lateral reaction with a non-linear characteristic, which operates upon the outer wheel where the wheelset has consumed the clearance on the track $\sigma$ (Lee and Cheng, 2005). The equation for such a force is

$$
Y_{\sigma j, j+1}=H\left(\left|y_{w j, j+1}-\zeta_{j, j+1}\right|-\frac{\sigma}{2}\right) \operatorname{sgn}\left(y_{w j, j+1}-\zeta_{j, j+1}\right) k_{y r}\left(\left|y_{w j, j+1}-\zeta_{j, j+1}\right|-\frac{\sigma}{2}\right)
$$

where $k_{y r}$ is the rail lateral stiffness and $H(\cdot)$ is Heaviside's unit step function. 


\section{The equations of motion of the vehicle}

The equations of lateral, roll and yaw motions of the vehicle carbody are written as below

$$
\begin{aligned}
& m_{c} \ddot{y}_{c}+c_{y c}\left[2\left(\dot{y}_{c}+h_{c} \dot{\varphi}_{c}\right)-\left(\dot{y}_{b 1}+\dot{y}_{b 2}\right)+h_{b 2}\left(\dot{\varphi}_{b 1}+\dot{\varphi}_{b 2}\right)\right] \\
& \quad+2 k_{y c}\left[2\left(y_{c}+h_{c} \varphi_{c}\right)-\left(y_{b 1}+y_{b 2}\right)+h_{b 2}\left(\varphi_{b 1}+\varphi_{b 2}\right)\right]=0 \\
& J_{x c} \ddot{\varphi}_{c}+2 c_{z c} b_{c}^{2}\left[2 \dot{\varphi}_{c}-\left(\dot{\varphi}_{b 1}+\dot{\varphi}_{b 2}\right)\right]+c_{y c} h_{c}\left[2\left(\dot{y}_{c}+h_{c} \dot{\varphi}_{c}\right)-\left(\dot{y}_{b 1}+\dot{y}_{b 2}\right)+h_{b 2}\left(\dot{\varphi}_{b 1}+\dot{\varphi}_{b 2}\right)\right] \\
& \quad+\left(k_{\varphi c}+2 k_{z c} b_{c}^{2}\right)\left[2 \varphi_{c}-\left(\varphi_{b 1}+\varphi_{b 2}\right)\right] \\
& \quad+2 k_{y c} h_{c}\left[2\left(y_{c}+h_{c} \varphi_{c}\right)-\left(y_{b 1}+y_{b 2}\right)+h_{b 2}\left(\varphi_{b 1}+\varphi_{b 2}\right)\right]-m_{c} g h_{c} \varphi_{c}=0 \\
& J_{z c} \ddot{\alpha}_{c}+2 c_{x c} b_{c}^{2}\left[2 \dot{\alpha}_{c}-\left(\dot{\alpha}_{b 1}+\dot{\alpha}_{b 2}\right)\right]+c_{y c} a_{c}\left[2 a_{c} \dot{\alpha}_{c}-\left(\dot{y}_{b 1}-\dot{y}_{b 2}\right)+h_{b 2}\left(\dot{\varphi}_{b 1}-\dot{\varphi}_{b 2}\right)\right] \\
& \quad+2 k_{x c} b_{c}^{2}\left[2 \alpha_{c}-\left(\alpha_{b 1}+\alpha_{b 2}\right)\right]+2 k_{y c} a_{c}\left[2 a_{c} \alpha_{c}-\left(y_{b 1}-y_{b 2}\right)+h_{b 2}\left(\varphi_{b 1}-\varphi_{b 2}\right)\right]=0
\end{aligned}
$$

where $m_{c}$ represents mass of the carbody, $J_{x c}$ is the inertia moment of the carbody around the longitudinal axis, and $J_{z c}$ - the inertia moment of the carbody around the vertical axis.

For $i=1,2$ and $j=2 i-1$, the equations of motion for the lateral displacement, roll and yaw of the bogies are as follows

$$
\begin{aligned}
& m_{b} \ddot{y}_{b i}+c_{y c}\left(\dot{y}_{b i}-h_{b 2} \dot{\varphi}_{b i}-\dot{y}_{c}-h_{c} \dot{\varphi}_{c} \mp a_{c} \dot{\alpha}_{c}\right)+2 c_{y b}\left[2\left(\dot{y}_{b i}+h_{b 1} \dot{\varphi}_{b i}\right)-\left(\dot{y}_{w j}+\dot{y}_{w(j+1)}\right)\right] \\
& \quad+2 k_{y c}\left(y_{b i}-h_{b 2} \varphi_{b i}-y_{c}-h_{c} \varphi_{c} \mp a_{c} \alpha_{c}\right)+2 k_{y b}\left[2\left(y_{b i}+h_{b 1} \varphi_{b i}\right)-\left(y_{w j}+y_{w(j+1)}\right)\right]=0 \\
& J_{x b} \ddot{\varphi}_{b i}+2 c_{z c} b_{c}^{2}\left(\dot{\varphi}_{b i}-\dot{\varphi}_{c}\right)+c_{y c} h_{b 2}\left(h_{b 2} \dot{\varphi}_{b i}-\dot{y}_{b i}+\dot{y}_{c}+h_{c} \dot{\varphi}_{c} \pm a_{c} \dot{\alpha}_{c}\right) \\
& \quad+2 c_{y b} h_{b 1}\left[2\left(h_{b 1} \dot{\varphi}_{b i}+\dot{y}_{b i}\right)-\left(\dot{y}_{w j}+\dot{y}_{w(j+1)}\right)\right]+4 c_{z b} b_{b}^{2} \dot{\varphi}_{b i}+\left(k_{\varphi c}+2 k_{z c} b_{c}^{2}\right)\left(\varphi_{b i}-\varphi_{c}\right) \\
& \quad+2 k_{y c} h_{b 2}\left(h_{b 2} \varphi_{b i}-y_{b i}+y_{c}+h_{c} \varphi_{c} \pm a_{c} \alpha_{c}\right) \\
& \quad+2 k_{y b} h_{b 1}\left[2\left(h_{b 1} \varphi_{b i}+y_{b i}\right)-\left(y_{w j}+y_{w(j+1)}\right)\right] \\
& \quad+\left[4 k_{z b} b_{b}^{2}-g\left(h_{12} \frac{m_{c}}{2}+h_{b 1} m_{b}\right)\right] \varphi_{b i}=0 \quad \text { with } \quad h_{12}=h_{b 1}+h_{b 2} \\
& J_{z b} \ddot{\alpha}_{b i}+2 c_{x c} b_{c}^{2}\left(\dot{\alpha}_{b i}-\dot{\alpha}_{c}\right)+2 c_{x b} b_{b}^{2}\left[2 \dot{\alpha}_{b i}-\left(\dot{\alpha}_{w j}+\dot{\alpha}_{w(j+1)}\right)\right] \\
& \quad+2 c_{y b} a_{b}\left[2 a_{b} \dot{\alpha}_{b i}-\left(\dot{y}_{w j}-\dot{y}_{w(j+1)}\right)\right]+2 k_{x c} b_{c}^{2}\left(\alpha_{b i}-\alpha_{c}\right) \\
& \quad+2 k_{x b} b_{b}^{2}\left[2 \alpha_{b i}-\left(\alpha_{w j}+\alpha_{w(j+1)}\right)\right]+2 k_{y b} a_{b}\left[2 a_{b} \alpha_{b i}-\left(y_{w j}-y_{w(j+1)}\right)\right]=0
\end{aligned}
$$

where $m_{b}$ represents suspended mass of the bogie, $J_{x b}$ is the inertia moment of the bogie chassis around the longitudinal axis and $J_{z b}$-inertia moment of the bogie chassis around the vertical axis.

For the wheelsets $j$ and $j+1$, the equations of lateral displacement and yaw motions and the equation for rotary motion around the wheelset axis with $i=1,2$ and $j=2 i-1$, are

$$
\begin{aligned}
& m_{w} \ddot{y}_{w j, j+1}+2 c_{y b}\left(\dot{y}_{w j, j+1}-\dot{y}_{b i}-h_{b 1} \dot{\varphi}_{b i} \mp a_{b} \dot{\alpha}_{b i}\right)+2 k_{y b}\left(y_{w j, j+1}-y_{b i}-h_{b 1} \varphi_{b i} \mp a_{b} \alpha_{b i}\right) \\
& \quad=Y_{j,(j+1) 1}+Y_{j,(j+1) 2}-Y_{\sigma j, j+1} \\
& J_{z w} \ddot{\alpha}_{w j, j+1}+2 c_{x b} b_{b}^{2}\left(\dot{\alpha}_{w j, j+1}-\dot{\alpha}_{b i}\right)+2 k_{x b} b_{b}^{2}\left(\alpha_{w j, j+1}-\alpha_{b i}\right) \\
& \quad+J_{y w} \frac{V}{r_{o}} \dot{\varphi}_{w j, j+1}=-e_{o}\left(X_{j,(j+1) 1}-X_{j,(j+1) 2}\right) \\
& J_{y w} \dot{\omega}_{w j, j+1}=-r_{o}\left(X_{j,(j+1) 1}+X_{j,(j+1) 2}\right)
\end{aligned}
$$

where $m_{w}$ is mass of the wheelset; $e_{o}, r_{o}$ - coordinates of the wheel-rail contact points when the wheelset is in the median position on the track; $J_{x w}, J_{y w}$ and $J_{z w}$-wheelset inertia moments. The term $J_{y w}\left(V / r_{o}\right) \dot{\varphi}_{w j, j+1}$ corresponds to the gyroscopic moment due to the combined effect of wheelset rotary motion around its own axis with roll motion. $X_{j,(j+1) 1,2}$ are longitudinal forces 
and $Y_{j,(j+1) 1,2}$ represent guidance forces acting upon the wheelsets $j$ and $j+1$, respectively, in the wheel/rail contact points 1 or 2 .

The above equations are added to the equations for bouncing and roll of the wheelsets, the motions that are dependent on the lateral displacement of the wheelset on the track. These equations are used to calculate the normal contact wheel/rail forces. When considering that the bounce coming from the lateral displacement of the wheelset on the track is very low (Wickens, 2005), the inertia effect of the wheelset mass in the vertical direction can be thus neglected. Hence, the balance equation for the vertical forces can be written as

$$
Q_{j,(j+1) 1}+Q_{j,(j+1) 2}=2 Q_{o}
$$

where $Q_{j,(j+1) 1,2}$ are vertical loads in the wheel/rail contact points and $Q_{o}$ is the static load acting on the wheel.

The equation for the wheelset roll motion is

$$
\begin{aligned}
& J_{x w} \ddot{\varphi}_{w j, j+1}-J_{y w} \frac{V}{r_{o}} \dot{\alpha}_{w j, j+1}-2 b_{b}^{2} c_{z b} \dot{\varphi}_{b i}-2 b_{b}^{2} k_{z b} \varphi_{b i} \\
& \quad=r_{o}\left(Y_{j,(j+1) 1}+Y_{j,(j+1) 2}\right)+e_{o}\left(Q_{j,(j+1) 1}-Q_{j,(j+1) 2}\right)+r_{o} Y_{\sigma j, j+1}
\end{aligned}
$$

where the term $J_{y w}\left(V / r_{o}\right) \dot{\alpha}_{w j, j+1}$ corresponds to the gyroscopic moment due to the combined effect of rotary motion of the wheelset around its own axis with the yaw motion.
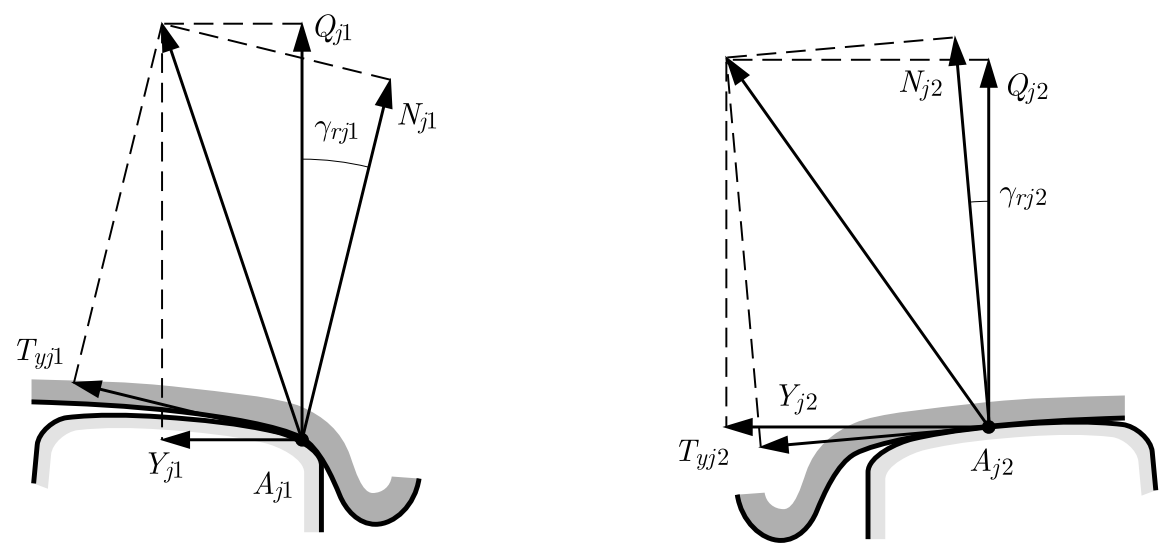

Fig. 3. he wheel/rail contact forces acting in the cross wheel-rail section

The wheel/rail contact forces, namely the longitudinal forces, guidance forces and the vertical loads are expressed as a function of the longitudinal components $T_{x j,(j+1) 1,2}$ and lateral $T_{y j,(j+1) 1,2}$ of the creep forces and the normal reactions $N_{j,(j+1) 1,2}$ in the wheel/rail contact points. As an example, the equations for the wheelsets $j$ (see Fig. 3) can be therefore written

$$
\begin{aligned}
& X_{j 1,2}=T_{x j 1,2} \\
& Y_{j 1,2}=T_{y j 1,2} \cos \gamma_{r j 1,2} \mp N_{j 1,2} \sin \gamma_{r j 1,2} \\
& Q_{j 1,2}= \pm T_{y j 1,2} \sin \gamma_{r j 1,2}+N_{j 1,2} \cos \gamma_{r j 1,2}
\end{aligned}
$$

where $\gamma_{r j 1,2}$ stand for the wheel/rail contact angles against the track reference system. Since the contact angles are small on the rolling surface areas, the equation below can be used

$$
\gamma_{r j 1,2}=\gamma_{o} \pm\left(y_{w j 1,2}-\zeta_{j}\right) \frac{1}{\rho_{w}-\rho_{r}} \frac{e_{o}+\rho_{w} \gamma_{o}}{e_{o}-r_{o} \gamma_{o}}
$$

where $\gamma_{o}$ is the wheel/rail contact angle for the median position of the wheelset on the track and $\rho_{w}$ and $\rho_{r}$ represent the curvature radii of the wheel-rail rolling profiles, respectively. 
According to Polach's friction nonlinear model (Polach, 1999), the components of creep forces have the following forms

$$
\begin{aligned}
& T_{x j 1,2}=-\frac{2 \mu N_{j 1,2}}{\pi}\left(\frac{\kappa_{j 1,2}}{1+\kappa_{j 1,2}^{2}}+\arctan \kappa_{j 1,2}\right) \frac{\nu_{x j 1,2}}{\nu_{c j 1,2}} \\
& T_{y j 1,2}=-\mu N_{j 1,2}\left\{\frac{2}{\pi}\left(\frac{\kappa_{j 1,2}}{1+\kappa_{j 1,2}^{2}}+\arctan \kappa_{j 1,2}\right) \frac{\nu_{y j 1,2}}{\nu_{c j 1,2}}\right. \\
&\left.+\frac{9}{16} a_{j 1,2} K_{M j 1,2}\left[1+6.3\left(1-\mathrm{e}^{(-a / b)_{j 1,2}}\right)\right] \frac{\nu_{s j 1,2}}{\nu_{c j 1,2}}\right\}
\end{aligned}
$$

where $\mu$ is the friction coefficient, $\nu_{x j 1,2}$ and $\nu_{y j 1,2}$ represent the longitudinal and the lateral components of the creepage in the wheel/rail contact points, $\nu_{c j 1,2}$ - creepage corrected by the spin, and $a_{j 1,2}$ and $b_{j 1,2}$ stand for the semiaxes of the contact ellipse.

To calculate the creepage in the wheel/rail contact points, the following equations are used

$$
\begin{aligned}
\nu_{x j 1,2} & =\mp \frac{y_{w j}-\zeta_{j}}{r_{o}} \gamma_{e}-\frac{\omega_{w j} r_{o}}{V} \mp \frac{e_{o} \dot{\alpha}_{w j}}{V} \\
\nu_{y j 1,2} & =\frac{1}{V}\left(\phi \dot{y}_{w j}-\lambda \dot{\zeta}_{j}\right)-\alpha_{w j} \quad \lambda=\frac{r_{o} \gamma_{o}}{e_{o}-r_{o} \gamma_{o}} \quad \phi=1+\lambda \\
\nu_{c j 1,2} & =\sqrt{\nu_{x j 1,2}^{2}+\nu_{y c j 1,2}^{2}}
\end{aligned}
$$

where

$$
\gamma_{e}=\frac{\rho_{r} \gamma_{o}}{\rho_{w}-\rho_{r}} \frac{e_{o}+\rho_{r} \gamma_{o}}{e_{o}-r_{o} \gamma_{o}}
$$

is the equivalent conicity and $\nu_{y c j 1,2}$ represents the lateral component of the creepage corrected by the spin.

In order to calculate $\nu_{y c j 1,2}$, the relations below are applied

$$
\nu_{y c j 1,2}=\left\{\begin{array}{lll}
\nu_{y j 1,2}+a_{j 1,2} \nu_{s j 1,2} & \text { for } & \left|\nu_{y j 1,2}+a_{j 1,2} \nu_{s j 1,2}\right|>\left|\nu_{y j 1,2}\right| \\
\nu_{y j 1,2} & \text { for } & \left|\nu_{y j 1,2}+a_{j 1,2} \nu_{s j 1,2}\right| \leqslant\left|\nu_{y j 1,2}\right|
\end{array}\right.
$$

where $\nu_{s j 1,2}$ is the spin defined as below

$$
\nu_{s j 1,2}=\frac{\dot{\alpha}_{w j}}{V} \mp\left(\frac{1}{r_{o}}+\frac{\omega_{w j}}{V}\right) \gamma_{w j 1,2}
$$

where $\gamma_{w j 1,2}$ represent the wheel/rail contact angles compared to the wheelset reference system

$$
\gamma_{w j 1,2}=\gamma_{o} \pm\left(y_{w j 1,2}-\zeta_{j}\right) \frac{1}{\rho_{w}-\rho_{r}} \frac{e_{o}+\rho_{r} \gamma_{o}}{e_{o}-r_{o} \gamma_{o}}
$$

To determine the semiaxes of the wheel/rail contact ellipse, the Hertz equations will be used as a function of the normal force and the curves of the rolling profiles.

As for the coefficient $\kappa_{j 1,2}$ in equations (3.8), it can be calculated from

$$
\kappa_{j 1,2}=\frac{1}{4} \frac{G \pi a_{j 1,2} b_{j 1,2} C_{i i}}{\mu N_{j 1,2}} \nu_{c j 1,2}
$$

where $G$ is the transverse elasticity modulus and $C_{i i}$ is a constant defined by Polach depending on the Kalker coefficients $C_{11}$ and $C_{22}$ (Kalker, 1967)

$$
C_{i i}=\sqrt{\left(C_{11} \frac{\nu_{x j 1,2}}{\nu_{j 1,2}}\right)^{2}+\left(C_{22} \frac{\nu_{y j 1,2}}{\nu_{j 1,2}}\right)^{2}} \quad \nu_{j 1,2}=\sqrt{\nu_{x j 1,2}^{2}+\nu_{y j 1,2}^{2}}
$$


The coefficient $K_{M j 1,2}$ in relation $(3.8)_{2}$ is

$$
K_{M j 1,2}=\left|\kappa_{s j 1,2}\right|\left(\frac{\delta_{j 1,2}^{3}}{3}-\frac{\delta_{j 1,2}^{2}}{2}+\frac{1}{6}\right)-\frac{1}{3} \sqrt{\left(1-\delta_{j 1,2}^{2}\right)^{3}}
$$

where

$$
\delta_{j 1,2}=\frac{\kappa_{s j 1,2}^{2}-1}{\kappa_{s j 1,2}^{2}+1} \quad \kappa_{s j 1,2}=\frac{8}{3} \frac{G b_{j 1,2} \sqrt{a_{j 1,2} b_{j 1,2}}}{\mu N_{j 1,2}} \frac{C_{23} \nu_{y c j 1,2}}{1+2 \pi\left[1-\mathrm{e}^{-(a / b)_{j 1,2}}\right]}
$$

while mentioning that $C_{23}$ is the coefficient calculated by Kalker (1967) for the spin.

The next step is calculation of the normal reactions $N_{j, 1,2}$ in the wheel/rail contact points of the wheelsets $j$. After some calculations, equations (3.4) and (3.5) become

$$
\begin{aligned}
& q_{j 1} N_{j 1}+q_{j 2} N_{j 2}=2 Q_{o} \\
& \left(r_{o} p_{j 1}-e_{o} q_{j 1}\right) N_{j 1}+\left(r_{o} p_{j 2}+e_{o} q_{j 2}\right) N_{j 2}=-c_{j}
\end{aligned}
$$

in which

$$
\begin{aligned}
& c_{j}=J_{x o} \ddot{\varphi}_{o j}-J_{y o} \frac{V}{r_{o}} \dot{\alpha}_{o j}-2 b_{b}^{2} c_{z b} \dot{\varphi}_{b i}-2 b_{b}^{2} k_{z b} \varphi_{b i}-r_{o} Y_{\sigma j} \\
& q_{j 1,2}=1 \mp\left(K_{j 1,2} \frac{\nu_{y j 1,2}}{\nu_{c j 1,2}}+K_{s j 1,2} \frac{\nu_{s j 1,2}}{\nu_{c j 1,2}}\right) \gamma_{r j 1,2} \\
& p_{j 1,2}=K_{j 1,2} \frac{\nu_{y j 1,2}}{\nu_{c j 1,2}}+K_{s j 1,2} \frac{\nu_{s j 1,2}}{\nu_{c j 1,2}} \pm \gamma_{r j 1,2}
\end{aligned}
$$

where

$$
\begin{aligned}
& K_{j 1,2}=\frac{2 \mu}{\pi}\left(\frac{\kappa_{j 1,2}}{1+\kappa_{j 1,2}^{2}}+\arctan \kappa_{j 1,2}\right) \\
& K_{s j 1,2}=\frac{9 \mu}{16} a_{j 1,2} K_{M j 1,2}\left[1+6.3\left(1-\mathrm{e}^{-(a / b)_{j 1,2}}\right)\right]
\end{aligned}
$$

It is worth mentioning that the set of equations (3.18) is non-linear because the coefficients $q_{j 1,2}$ and $p_{j 1,2}$ depend on the normal forces $N_{j 1,2}$. The solution to the set can be derived by simple iterations via the equations

$$
N_{j 1}=\frac{2\left(r_{o} p_{j 2}+e_{o} q_{j 2}\right) Q_{o}+q_{j 2} c_{j}}{r_{o}\left(q_{j 1} p_{j 2}-q_{j 2} p_{j 1}\right)+2 e_{o} q_{j 1} q_{j 2}} \quad N_{j 2}=\frac{-q_{j 1} c_{j}-2\left(r_{o} p_{j 1}-e_{o} q_{j 1}\right) Q_{o}}{r_{o}\left(q_{j 1} p_{j 2}-q_{j 2} p_{j 1}\right)+2 e_{o} q_{j 1} q_{j 2}}
$$

Equations of motion (3.1)-(3.3) can be solved by the Runge-Kutta method and relations (3.21) are used iteratively for each integration step in the calculation of the wheel/rail contact normal forces.

\section{Numerical analysis}

This Section features the results of numerical simulations regarding the influence of parameters of the lateral suspension on ride quality in the railway vehicle at velocity of $200 \mathrm{~km} / \mathrm{h}$ on a track with lateral irregularities. The evaluation of ride quality is based on the lateral R.M.S. acceleration, calculated in three reference points on the carbody longitudinal axis and at the floor level - at the carbody centre and above the two bogies.

The parameters of the vehicle numerical model are shown in Table 1. To calculate geometrical parameters of the wheel/rail contact, the S78 profile wheel and the UIC 60 rail with the standard 
Table 1. Parameters of the numerical model

\begin{tabular}{|l|l|}
\hline$m_{c}=34000 \mathrm{~kg}$ & $2 c_{x c}=50 \mathrm{kNs} / \mathrm{m}$ \\
\hline$m_{b}=3200 \mathrm{~kg}$ & $c_{y c}=15.205 \mathrm{kNs} / \mathrm{m}$ \\
\hline$m_{w}=1650 \mathrm{~kg}$ & $2 c_{z c}=34.44 \mathrm{kNs} / \mathrm{m}$ \\
\hline$J_{x c}=57460 \mathrm{kgm}^{2}$ & $2 k_{x c}=340 \mathrm{kN} / \mathrm{m}$ \\
\hline$J_{z c}=2456500 \mathrm{kgm}^{2}$ & $2 k_{y c}=340 \mathrm{kN} / \mathrm{m}$ \\
\hline$J_{x b}=3200 \mathrm{kgm}^{2}$ & $2 k_{z c}=1.2 \mathrm{MN} / \mathrm{m}$ \\
\hline$J_{z b}=5000 \mathrm{kgm}^{2}$ & $k_{\varphi c}=10 \mathrm{kNm}$ \\
\hline$J_{x w}=J_{z w}=928.125 \mathrm{kgm}^{2}$ & $4 c_{x b}=100 \mathrm{kNs} / \mathrm{m}$ \\
\hline$J_{y w}=349.14 \mathrm{kgm}^{2}$ & $4 c_{y b}=35.77 \mathrm{kNs} / \mathrm{m}$ \\
\hline $2 a_{c}=19 \mathrm{~m}$ & $4 c_{z b}=52.21 \mathrm{kNs} / \mathrm{m}$ \\
\hline $2 a_{b}=2.56 \mathrm{~m}$ & $4 k_{x b}=140 \mathrm{MN} / \mathrm{m}$ \\
\hline$h_{c}=1.3 \mathrm{~m}$ & $4 k_{y b}=10 \mathrm{MN} / \mathrm{m}$ \\
\hline$h_{b 1}=0.25 \mathrm{~m} ; h_{b 2}=0.2 \mathrm{~m}$ & $4 k_{z b}=4.4 \mathrm{MN} / \mathrm{m}$ \\
\hline $2 b_{b}=2 b_{c}=2 \mathrm{~m}$ & $k_{y r}=100 \mathrm{MN} / \mathrm{m}$ \\
\hline$\sigma=12 \mathrm{~mm}$ & $\mu=0.36$ \\
\hline
\end{tabular}

(a)

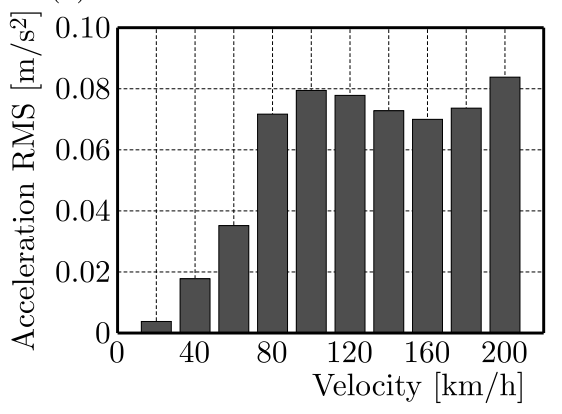

(b)

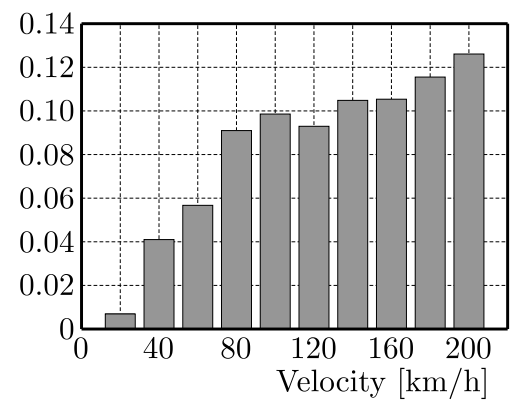

(c)

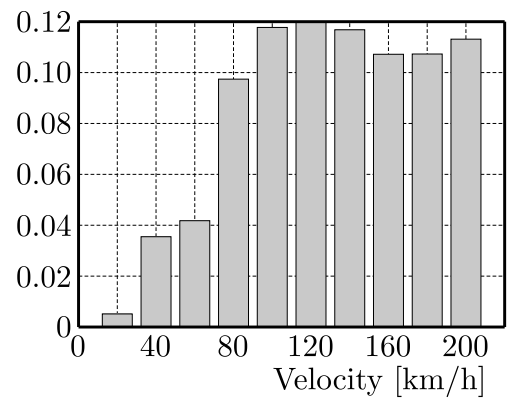

Fig. 4. The lateral R.M.S. acceleration of the carbody: (a) at the carbody centre, (b) above the front bogie, (c) above the rear bogie

C.F.R. of $1 / 20$ are considered. When the wheelset occupies the median position on the track with the normal gauge, the coordinates of the wheel/rail contact points are $r_{o}=0.4598 \mathrm{~m}$, $e_{o}=0.754 \mathrm{~m}$ and $\gamma_{o}=0.0495$, and the profiles radii are: $\rho_{w}=500 \mathrm{~mm}$ and $\rho_{r}=300 \mathrm{~mm}$ (Dumitriu, 2013). The equivalent conicity is $\gamma_{e}=0.1237$.

For the reference parameters of the numerical model presented in Table 1, Fig. 4 shows the lateral R.M.S. acceleration calculated in the three reference points of the carbody, ranging from 20 to $200 \mathrm{~km} / \mathrm{h}$. The velocity increases along with the lateral acceleration, which is not uniform due to the geometrical filtering effect coming from the vehicle wheelbases. Further on, it should be noted that, irrespective of the velocity, the acceleration is lower at the carbody centre and rises against the bogies. Also, it exhibits different behaviour of the vehicle in the reference points located above the two bogies. Should the carbody critical point in terms of vibrations is that reference point, where the acceleration is higher, this point can be identified against either the front bogie or the rear bogie, depending on the velocity. Thus, the carbody critical point is found above the front bogie up to velocity of $60 \mathrm{~km} / \mathrm{h}$. When the speed increases, the critical point migrates over the rear bogie - in this reference point, the carbody acceleration has the maximum value for velocities going from 80 to $160 \mathrm{~km} / \mathrm{h}$. When the vehicle travels at a speed of 180 or $200 \mathrm{~km} / \mathrm{h}$, the reference point above the front bogie is the carbody critical point. 
Figure 5 shows the carbody lateral R.M.S. acceleration of the vehicle at the velocity of $200 \mathrm{~km} / \mathrm{h}$ for values of the secondary suspension lateral stiffness ranging from $10^{4} \mathrm{~N} / \mathrm{m}$ to 5 . $10^{5} \mathrm{~N} / \mathrm{m}$. The higher $k_{y c}$, the worse ride quality is, a fact also visible in the continuous increase of the acceleration. For instance, for the reference value of the lateral stiffness, the R.M.S. acceleration is $0.084 \mathrm{~m} / s^{2}$ - at the carbody centre; $0.126 \mathrm{~m} / \mathrm{s}^{2}$ - above the front bogie; $0.113 \mathrm{~m} / \mathrm{s}^{2}$ - above the rear bogie. If $k_{y c}$ goes up to $5 \cdot 10^{5} \mathrm{~N} / \mathrm{m}$, the following values of acceleration will be obtained: $0.237 \mathrm{~m} / \mathrm{s}^{2}$ - at the carbody centre; $0.329 \mathrm{~m} / \mathrm{s}^{2}$ - above the front bogie; $0.363 \mathrm{~m} / \mathrm{s}^{2}-$ above the rear bogie. It is an increase of about circa three times of the lateral acceleration at the vehicle carbody level.

The diagrams in Fig. 5 also reveal an interesting fact, namely that for a rise in the lateral stiffness of the secondary suspension, the carbody critical point migrates from one reference point to another. Thus, for $k_{y c}$ ranging from $10^{4} \mathrm{~N} / \mathrm{m}$ to $1.7 \cdot 10^{5} \mathrm{~N} / \mathrm{m}$, the critical point is located above the front bogie, while for $k_{y c}$ as $5 \cdot 10^{5} \mathrm{~N} / \mathrm{m}$, this point is the carbody reference point against the rear bogie.

(a)

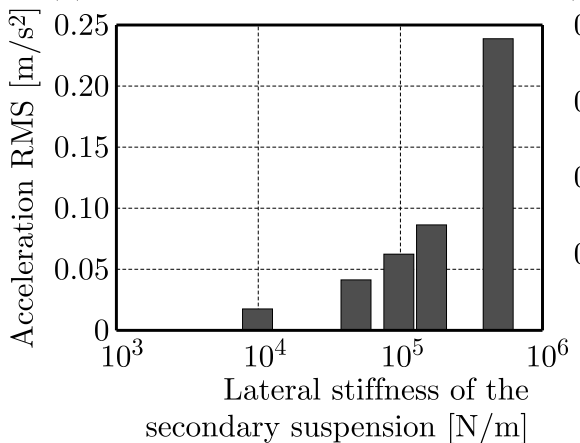

(b)

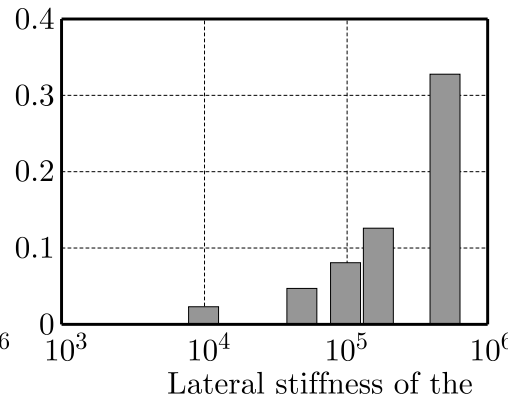

secondary suspension $[\mathrm{N} / \mathrm{m}]$ (c)

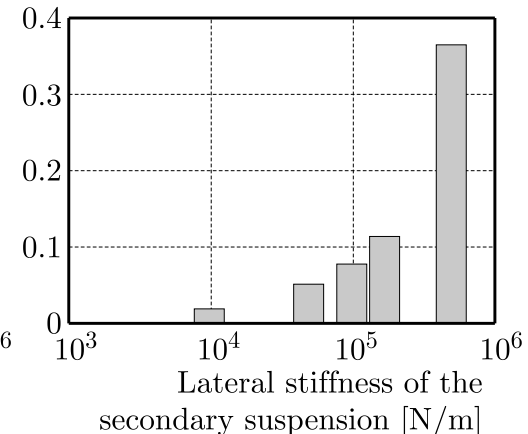

Fig. 5. The influence of the lateral stiffness of the secondary suspension upon ride quality: (a) at the carbody centre, (b) above the front bogie, (c) above the rear bogie

(a)

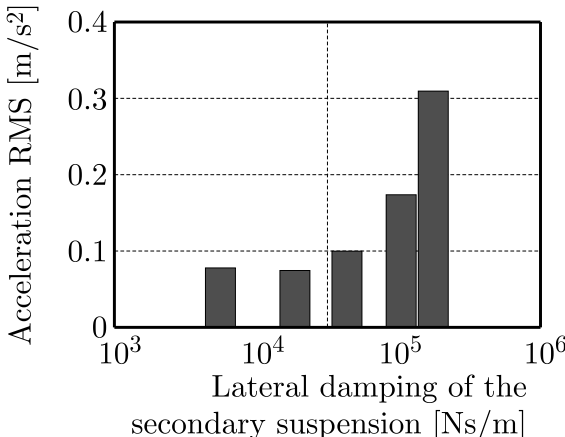

(b)

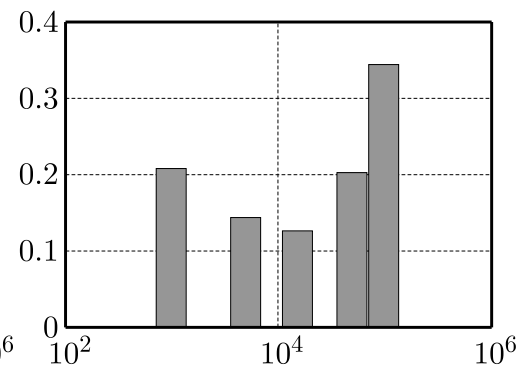

Lateral damping of the secondary suspension $[\mathrm{Ns} / \mathrm{m}]$ (c)

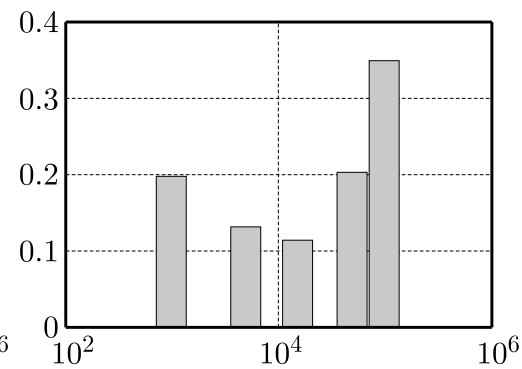

Lateral damping of the secondary suspension $[\mathrm{Ns} / \mathrm{m}]$

Fig. 6. The influence of the lateral damping of the secondary suspension upon ride quality: (a) at the carbody centre, (b) above the front bogie, (c) above the rear bogie

Upon examining the diagrams in Fig. 6, the observation is that there is a value of the lateral damping of the secondary suspension for which the lateral acceleration in any of the carbody reference points has the minimum value. At the carbody centre, where the level of vibrations is lower, the minimum acceleration is calculated for $c_{y c}=5 \cdot 10^{3} \mathrm{Ns} / \mathrm{m}$. On the other hand, the same thing happens above the bogies for higher values of the lateral damping, which is here the reference value itself $\left(c_{y c}=1.52 \cdot 10^{4} \mathrm{Ns} / \mathrm{m}\right)$. The conclusion is that an increase in the lateral damping of the secondary suspension up to a certain value generally improves ride quality. Over this limit, the damping has a contrary effect, evident in a significant rise of the 
R.M.S. acceleration. For instance, this acceleration is $0.074 \mathrm{~m} / \mathrm{s}^{2}$ for $c_{y c}=5 \cdot 10^{3} \mathrm{Ns} / \mathrm{m}$ at the carbody centre. When raising the lateral damping up to $10^{5} \mathrm{Ns} / \mathrm{m}$, the acceleration becomes $0.31 \mathrm{~m} / \mathrm{s}^{2}$. The same thing occurs in the reference points above the bogies. Thus, above the front bogie, the R.M.S. acceleration is $0.126 \mathrm{~m} / s^{2}$ for $c_{y c}=1.52 \cdot 10^{4} \mathrm{Ns} / \mathrm{m}$; when $c_{y c}$ reaches $10^{5} \mathrm{Ns} / \mathrm{m}$, the acceleration goes up to $0.345 \mathrm{~m} / \mathrm{s}^{2}$. Another observation is related to the fact that the lateral damping of the secondary suspension influences the position of the critical point of the carbody. In this case, it is about its migration from the front bogie to the rear bogie, when $c_{y c}$ is $10^{5} \mathrm{Ns} / \mathrm{m}$.

The diagrams in Fig. 7 help with further examination of the influence of the lateral stiffness of the primary suspension upon ride quality, thus considering values of $k_{y b}$ between $10^{6} \mathrm{~N} / \mathrm{m}$ and $5 \cdot 10^{7} \mathrm{~N} / \mathrm{m}$. For $k_{y b}=5 \cdot 10^{6} \mathrm{~N} / \mathrm{m}$, the lateral R.M.S. acceleration calculated in the carbody reference points has the minimum value: $0.080 \mathrm{~m} / \mathrm{s}^{2}$ - at the carbody centre; $0.121 \mathrm{~m} / \mathrm{s}^{2}$ - against the front bogie; $0.106 \mathrm{~m} / \mathrm{s}^{2}$ - against the rear bogie. On the other hand, for the above cases, a change in the lateral stiffness of the primary suspension does not lead to the movement of the carbody critical point, which remains above the front bogie.

(a)

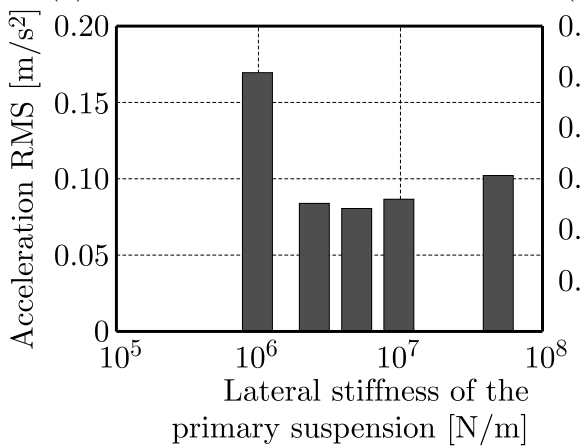

(b)

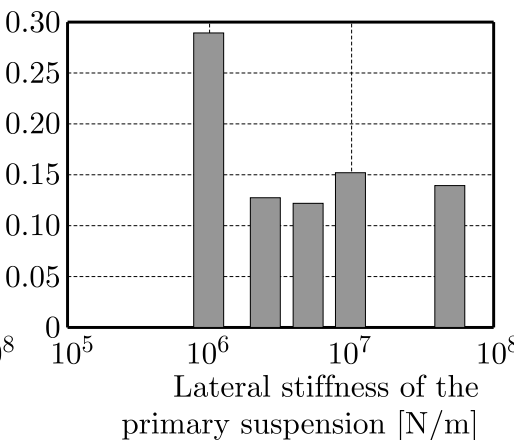

(c)

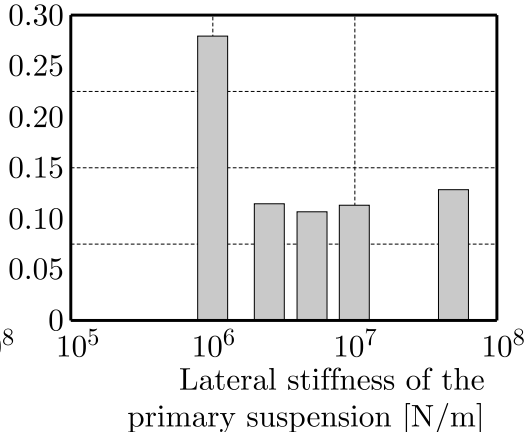

Fig. 7. The influence of the lateral stiffness of the primary suspension upon ride quality: (a) at the carbody centre, (b) above the front bogie, (c) above the rear bogie

(a)

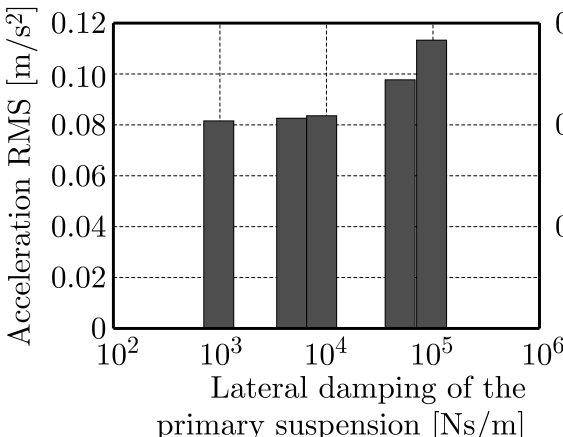

(b)

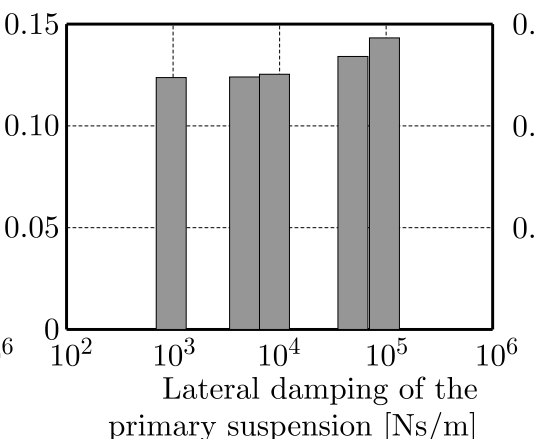

(c)

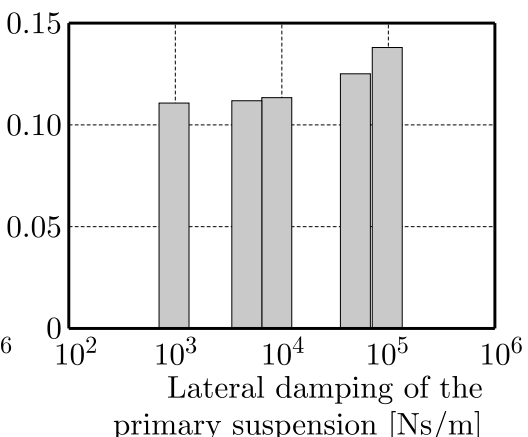

Fig. 8. The influence of the lateral damping of the primary suspension upon ride quality: (a) at the carbody centre, (b) above the front bogie, (c) above the rear bogie

The influence of lateral damping of the primary suspension upon ride quality is evident in the diagrams in Fig. 8. They include the results regarding the lateral R.M.S. acceleration for values of $c_{y b}$ within the interval of $10^{3}, 10^{4}, 10^{5} \mathrm{Ns} / \mathrm{m}$. It can observed that lateral damping of the primary suspension does not have a significant influence upon the R.M.S. acceleration. However, for better ride quality, it is desirable to have the lowest possible lateral damping of the primary suspension. To validate this hypothesis, let us look at the results corresponding to the limit values of the interval defined for $c_{y b}$. Thus, for $c_{y b}=10^{3} \mathrm{Ns} / \mathrm{m}$, the R.M.S. acceleration in the 
carbody reference points is $0.081 \mathrm{~m} / s^{2}$ - at the carbody centre; $0.124 \mathrm{~m} / \mathrm{s}^{2}$ - above the front bogie; $0.111 \mathrm{~m} / \mathrm{s}^{2}-$ above the rear bogie. For $c_{y b}=10^{5} \mathrm{Ns} / \mathrm{m}$, the results are as such: $0.113 \mathrm{~m} / \mathrm{s}^{2}$ - at the carbody centre; $0.144 \mathrm{~m} / \mathrm{s}^{2}$ - above the front bogie; $0.138 \mathrm{~m} / \mathrm{s}^{2}$ - above the rear bogie. As for the position of the carbody critical point, it can be proven that this corresponds to the reference point above the first bogie, irrespective of the value of $c_{y b}$ being considered.

\section{Conclusions}

The paper hereby examines the influence of suspension parameters upon ride quality compared to lateral vibrations of a railway vehicle during running on a track with lateral irregularities. The ride quality is evaluated by numerical simulations, based on the lateral accelerations in three reference points of the carbody - at its centre and above the bogies.

The results thus obtained validate, on one hand, some features of lateral vibrations of the railway vehicle and, on the other hand, the possibility to improve ride quality by the best possible selection of the lateral suspension parameters.

It has been shown that, irrespective of the velocity, the lateral acceleration is lower at the carbody centre and higher above the bogies. Similarly, the asymmetry of the carbody vibrations has been noticed in the reference points against the two bogies. The concept of the carbody critical point in terms of the vibration level has been introduced as being that reference point where the acceleration has the highest value. Its position depends on velocity and parameters of the secondary suspension.

The analysis of the influence of the suspension parameters on the carbody lateral acceleration has confirmed that the most appropriate values of the lateral damping in the secondary suspension or of the lateral stiffness in the primary suspension can be identified. For these values, the carbody acceleration can be lowered to minimum, hence enabling the vehicle to have the best dynamic performance in terms of ride quality. Likewise, the lateral stiffness of the secondary suspension can significantly affect ride quality under certain conditions.

\section{References}

1. Cheng Y.C., Leeb, S.Y., Chen, H.H., 2009, Modeling and nonlinear hunting stability analysis of high-speed railway vehicle moving on curved track, Journal Sound and Vibration, 324, 139-160

2. Dabin C., Li L., Jin X., Xiao X., Ding J., 2012, Influence of vehicle parameters on critical hunting speed based on Ruzicka model, Chinese Journal of Mechanical Engineering, 25, 1, 536-542

3. Dumitriu M., 2013, Modelling the geometric contact between wheels and the rails of a track with horizontal irregularity, Mechanical Journal Fiability and Durability, 1, 116-122

4. Dumitriu M., 2014, On-line running tests for validating the numerical simulations of the vertical dynamic behavior in railway vehicles, Applied Mechanics and Materials, 657, 609-613

5. Evans J., Berg M., 2009, Challenges in simulation of rail vehicle dynamics, Vehicle System Dynamics, 47, 1023-1048

6. Garg V.K., Dukkipati R.V., 1984, Dynamics of Railway Vehicle Systems, Academic Press, New York

7. He Y., McPhee J., 2002, Optimization of the lateral stability of rail vehicles, Vehicle System Dynamics, 38, 5, 361-390

8. Huang C., Zeng J., Liang S., 2013, Carbody hunting investigation of a high speed passenger car, Journal of Mechanical Science and Technology, 27, 8, 2283-2292

9. Iwnicki S., 2006, Handbook of Railway Vehicle Dynamics, CRC Press Taylor \& Francis Group 
10. Kalker J.J., 1967, On the rolling contact of two elastic bodies in the presence of dry friction, Ph.D. Thesis, Delft

11. Lee S.Y., Cheng Y.C., 2005, Hunting stability analysis of high-speed railway vehicle trucks on tangent tracks, Journal of Sound and Vibration, 282, 881-898

12. Mazzola L., Alfi S., Bruni S., 2010, A method to optimize stability and wheel wear in railway bogies, International Journal of Railway, 3, 3, 95-105

13. ORE B 176, 1989, Bogies with steered or steering wheelsets, Report No. 1: Specifications and preliminary studies, Vol.2, Specification for a bogie with improved curving characteristics

14. Park J.P., Кон H.I., Kim N.P., 2011, Parametric study of lateral stability for a railway vehicle, Journal of Mechanical Science and Technology, 25, 7, 1657-1666

15. Polach O., 1999, A fast wheel-rail forces calculation computer code, Vehicle System Dynamics, 33, 728-739

16. Polach O., 2006, On non-linear methods of bogie stability assessment using computer simulations, Proceedings of the Institution of Mechanical Engineers, Part F: Journal of Rail and Rapid Transit, 220, 13-27

17. Polach O., Kaiser I., 2012, Comparison of methods analyzing bifurcation and hunting of complex rail vehicle models, Journal of Computational and Nonlinear Dynamics, 7, 041005-1-041005-8

18. Schupp G., 2003, Simulation of railway vehicles: Necessities and applications, Mechanics Based Design of Structures and Machines, 31, 3, 297-314

19. Serajian R., 2013, Parameters' changing influence with different lateral stiffness on nonlinear analysis of hunting behavior of a bogie, Vibroengineering. Journal of Measurements in Engineering, 1, 4, 195-206

20. Sharma R.C., 2011, Parametric analysis of rail vehicle parameters influencing ride behavior, International Journal of Engineering, Science and Technology, 3, 8, 54-65

21. Suarez B., Mera J.M. , Martinez M.L. Jose, Chover J.A., 2013, Assessment of the influence of the elastic properties of rail vehicle suspensions on safety, ride quality and track fatigue, Vehicle System Dynamics, 51, 2, 280-300

22. UIC 518 Leaflet, 2009, Testing and approval of railway vehicles from the point of view of their dynamic behaviour - Safety - Track Fatigue - Ride Quality

23. Wickens A.H., 2005, Fundamentals of rail vehicles dynamics: guidance and stability, Taylor \& Francis e-Library

24. Zboinski K., Dusza M., 2010, Self-exciting vibrations and Hopf's bifurcation in non-linear stability analysis of rail vehicles in a curved track, European Journal of Mechanics - A/Solids, 29, 190-203

25. Zboinski K., Dusza M., 2011, Extended study of railway vehicle lateral stability in a curved track, Vehicle System Dynamics, 49, 789-810 